\title{
DRUG UTILIZATION ANALYSIS OF ANTI-SNAKE VENOM AT A TERTIARY CARE CENTER IN CENTRAL MAHARASHTRA: A 3-YEAR RETROSPECTIVE STUDY
}

\author{
KETAN Y ASAWALE ${ }^{1}$, MUKESH C MEHTA ${ }^{2}$, PRAVIN S UIKE ${ }^{3 *}$ \\ ${ }^{1}$ Department of Clinical Pharmacology, Seth GS Medical College \& KEM Hospital, Mumbai - 400 012, Maharashtra, India. ${ }^{2}$ Department \\ of Pharmacology, Indira Gandhi Government Medical College, Nagpur - 440 018, Maharashtra, India. ${ }^{3}$ Department of Pharmacology, \\ Government Medical College, Akola - 444 001, Maharashtra, India. Email: pravin_vike@yahoo.co.in
}

Received: 23 March 2018, Revised and Accepted: 23 April 2018

ABSTRACT

Objectives: In India, snakebite is a major public health problem. Anti-snake venom serum (ASVS) is the definitive treatment for poisonous snakebites. However, the non-availability of effective ASVS to treat the specific types of snakebite envenomation encountered in various regions of the world has become a critical health issue at global level. In the present observational descriptive study, we observed the prescribing pattern and rationality of ASVS and the dosage of ASVS at our hospital and compared them with the standard guidelines in the treatment of snakebite cases with the aim to observe optimum utilization of ASVS.

Methods: Data collection of registered cases of snakebite of 3 years from January to December 2012, 2013, and 2014 was carried out between January 2015 and December 2015.

Results: A total of 935 cases were studied. ASVS treatment was given to 774 cases of snakebite, out of which 161 had no bite marks and 613 patients had bite marks. Majority (615 [79.45\%]) of cases were treated with 1-20 vials of ASVS during total stay at center. Overall, 899 cases were discharged, among them 606 cases were not followed the World Health Organization guidelines. 29 cases succumbed and seven were referred to other center. The mortality observed during the 3 years was $3.10 \%$.

Conclusion: We observed individualized treatment for treatment with ASVS, thereby reducing the use of ASVS. Thus, we conclude that individualized approach depending on locally predominant snakes for the treatment of snakebite based on the degree of envenomation is a way by which the scarce ASVS can be used optimally.

Keywords: Anti-snake venom serum, Envenomation, Prescribing patterns, Snakebite, World Health Organization guidelines.

(C) 2018 The Authors. Published by Innovare Academic Sciences Pvt Ltd. This is an open access article under the CC BY license (http://creativecommons. org/licenses/by/4. 0/) DOI: http://dx.doi.org/10.22159/ajpcr.2018.v11i8.26174

\section{INTRODUCTION}

Snakes inhabit the entire globe except a few islands such as the Arctic, New Zealand, and Ireland. The temperate and tropical countries are the ones with most species and number of snakes [1]. Snakebite is a lifethreatening medical emergency, frequently occurring among rural people, especially those working in the fields. Snakebites were not considered as a major health problem, but the World Health Organization (WHO) has added snakebites in the list of neglected tropical diseases in 2009 [1,2].

In India, snakebite is a major public health problem. As per survey conducted by Warrel et al., the annual incidence of snakebite is 66-163 per 1 lakh population. The morbidity and mortality rates are 1.4-68 and 1.1-2.4 per 1 lakh population, respectively. The survey also reports that the case fatality rate is $1.7 \%-20 \%$ and estimates that $35,000-50,000$ people die of snakebite in India each year [2]. Bhalla et al., in 2014 [3-6], reported that the states with a large number of snakebite cases are Maharashtra, West Bengal, Tamil Nadu, Uttar Pradesh, and Kerala. Alirol et al. in a review article in 2010 stated that in Maharashtra about 2000 deaths occur every year due to snakebite. The incidence of snakebites is common in areas with hot and dry climate of Konkan and Vidarbha region of Maharashtra $[2,3,7,8]$. A recent reported incidence of snakebite cases in our institution is $0.6 \%$, which is one of the highest in Maharashtra [9].

As per guidelines given by the WHO in 2010 [1], anti-snake venom serum (ASVS) is the definitive treatment for poisonous snakebites. The production of anti-snake involves processes from selection of poisonous snake, acquisition of venom, processing of animal, quality control of production, clinical trial of ASVS, and mass scale production and distribution $[10,11]$. Due to this long process and animal ethics issues involved in the production of ASVS, it has become very difficult to provide ASVS at the correct place at the correct time. The unavailability of effective ASVS to treat the specific types of snakebite envenomation encountered in various regions of the world has become a critical health issue at global level. The crisis has reached its greatest intensity in Southeast Asia, and India is also suffering from a lack of effective and affordable products $[5,6]$.

In a study conducted by Whitekar et al., in 2008 [10], it was found that only $20 \%$ of snakebites result in significant envenomation that requires ASVS therapy. Species wise 50\% Russell's viper bites, 30\% cobra bites, and $5-10 \%$ saw-scaled viper bites do not require ASVS [12]. However, snakebite is a life-threatening emergency that comes under medicolegal cases, and hence, there might be over prescribing, as the physicians might not want to take risk in treatment. Thus, even though there are guidelines provided by the WHO, there is a deviation from the protocol for treatment when it comes to doses of ASVS.

Keeping all these facts in mind, the present study was conducted to observe the prescribing pattern and rationality of ASVS. We also intend to compare the dosage of ASVS at our hospital with standard guidelines in the treatment of snakebite cases with the aim to observe optimum utilization of ASVS. This will help conserve ASVS.

\section{METHODS}

The present study was an observational descriptive study. The data collection of registered cases of snakebite of 3 years from January to 
December 2012, 2013, and 2014 was carried out between January 2015 and December 2015 from medical records section. Due permission from institutional ethics committee (IEC) was taken before the commencement of the study. A total of 935 cases were studied from the medical records department (MRD) of the tertiary care center, and data were recorded on predefined case record forms (CRFs).

All registered cases of snakebite in the tertiary care center from January to December 2012, 2013 and 2014. Data were collected in predesigned CRFs prepared for this study. The CRF was submitted to IEC and MRD for approval. The work of data collection from registered indoor patient forms was done in the MRD under surveillance. Prescriptions were evaluated for demographic data, ASVS prescribing information, and WHO core drug prescription indicators. Demographics were analyzed using descriptive statistics. Categorical data were expressed as proportion and numerical data were expressed as median with interquartile range. Statistical analysis was done using Microsoft Office Excel ${ }^{\circledR} 2007$.

\section{RESULTS}

The present study enrolled a total of 935 cases of snakebite between January 2012 and December 2014. Table 1 summarizes the demographic profile of the patients. From this Table 1, it was observed that the maximum number of cases $(80.74 \%)$ of snakebite was from the age groups of 15 to 59 years.

About $94.97 \%$ cases of snakebites were observed before the administration of ASVS, whereas only 5.03\% cases were given ASVS without observation (Table 2). It was observed that 137 cases of severe envenomation, 128 (93.43\%) cases were observed for signs of envenomation before administration of ASVS, and remaining nine received ASVS without observation. Similarly, out of 446 cases with mild envenomation, 410 (91.95\%) were observed before giving ASVS, while 36 cases received ASVS without observation (Table 2). Table 3 summarizes the relation between the degree of envenomation and ASVS administration.

$417(44.59 \%)$ cases out of 935 cases received ASVS in the range of 1-10 vials during the duration of treatment and 198 (21.17\%) patients received 11-20 vials of ASVS (Table 4). Similarly, 77 (8.23\%) cases were given $21-30$ vials, 48 (5.13\%) were administered $31-40$ vials, $30(3.2 \%)$ received $41-50$ vials, and $4(0.4 \%)$ cases received $51-60$ vials. However, 161 (17.21\%) patients out of 935 did not receive any ASVS. In the present study, the average quantity of ASVS administered in cases with mild envenomation was 11.91 vials and in severe envenomation cases were 23.93 vials. Table 5 summarizes the relationship between bite marks with administration of ASVS.

In the present study, out of total (935) cases, 899 cases were discharged. $352(39.15 \%)$ cases out of the discharged cases had no signs of envenomation, 440 (48.94\%) had mild signs of envenomation, and $107(11.90 \%)$ cases had severe degree of envenomation. Similarly, $29(3.10 \%)$ cases out of 935 succumbed to snakebite and all cases were of severe envenomation. 7 ( $0.74 \%)$ out of 935 cases of snakebite were referred to other centers, and of these, six had mild signs of envenomation and one had severe signs of envenomation (Table 6). Of 625 cases where the WHO guidelines were not followed, 606 were discharged, 16 succumbed, and three were referred. Among 149 cases out of 935 where the WHO guidelines were followed, 132 were discharged, 13 died, and four were referred (Table 7).

\section{DISCUSSION}

Snakebite is a common health hazard in India. The incidence of snakebite cases has been dormant in the past few years but now is on the increase due to better reporting and better accessibility to medical care centers and expanding source of disease information through the sources such as journals and internet. ASVS is the definitive treatment for poisonous snakebites [5,6]. Limitations to the use of ASVS are because ASVS is difficult to acquire nowadays due to many reasons such as ever-changing regulations in quality control, animal ethics issues in production, and slow production processes [13]. Since snakebites are emergency conditions, the patients and relatives are in an anxious state in the emergency department. Furthermore, there are chances that the patient has already taken a dose of ASVS at the peripheral center, but the physician was not informed about it. Thus, due to the panic states and inadequate information by the patient, the physicians have a big problem to decide the dose of ASVS. Literature search shows that there is no standardized dose of ASVS for the treatment of snakebites. The dose of ASVS needed to treat snakebites depends on many factors such as species of the snake, type of bite, intraspecies variation, and degree of envenomation.

Table 1: Demographic data of the patients

\begin{tabular}{ll}
\hline Demographic data & Number of patients \\
\hline Age group (in years) & \\
$0-14$ & $98(10.48)$ \\
$15-29$ & $302(32.30)$ \\
$30-44$ & $289(30.91)$ \\
$45-59$ & $164(17.54)$ \\
$60-74$ & $70(1.28)$ \\
Gender & \\
Male & $526(56.25)$ \\
Female & $408(43.75)$ \\
\hline
\end{tabular}

Table 2: Relation between degree of envenomation and observation of patient before treating with ASVS

\begin{tabular}{llll}
\hline \multirow{2}{*}{ Degree of envenomation } & \multicolumn{2}{c}{ Patient under observation } & \multirow{2}{*}{ Total } \\
\cline { 2 - 3 } & No & Yes & \\
\hline None & 2 & 350 & 352 \\
Mild & 36 & 410 & 446 \\
Sever & 9 & 128 & 137 \\
Total & 47 & 888 & 935 \\
\hline
\end{tabular}

ASVS: Anti-snake venom serum

Table 3: Relation between degree of envenomation and ASVS administration

\begin{tabular}{llll}
\hline \multirow{2}{*}{ Degree of envenomation } & \multicolumn{2}{c}{ ASVS treatment } & \multirow{2}{*}{ Grand total } \\
\cline { 2 - 3 } & Not given & Given & \\
\hline None & 161 & 191 & 352 \\
Mild & 0 & 446 & 446 \\
Severe & 0 & 137 & 137 \\
Total & 161 & 774 & 935 \\
\hline
\end{tabular}

ASVS: Anti-snake venom serum

Table 4: Doses of ASVS in snakebite cases

\begin{tabular}{lll}
\hline Signs of envenomation & Mean dose of ASVS & Mode \\
\hline None & 27.21 & 0 \\
Mild & 119.06 & 30 \\
Severe & 239.27 & 190 \\
\hline
\end{tabular}

ASVS: Anti-snake venom serum

Table 5: Relation between bite marks and ASVS treatment

\begin{tabular}{llll}
\hline Bite marks & \multicolumn{2}{c}{ ASVS treatment } & \multirow{2}{*}{ Total } \\
\cline { 2 - 3 } & Not given & Given & \\
\hline Not seen & 100 & 161 & 261 \\
Seen & 61 & 613 & 674 \\
Total & 161 & 774 & 935 \\
\hline
\end{tabular}

ASVS: Anti-snake venom serum 
Table 6: Patient outcome with respect to the degree of envenomation

\begin{tabular}{lllll}
\hline \multirow{2}{*}{$\begin{array}{l}\text { Degree of } \\
\text { envenomation }\end{array}$} & \multicolumn{2}{l}{ Patient outcome } & \multirow{2}{*}{ Total } \\
\cline { 2 - 4 } & Discharged & Death & Referred & \\
\hline None & 352 & 0 & 0 & 352 \\
Mild & 440 & 0 & 6 & 446 \\
Severe & 107 & 29 & 1 & 137 \\
Total & 899 & 29 & 7 & 935 \\
\hline
\end{tabular}

Table 7: Relation of patient outcome with following of guidelines of treatment at tertiary center

\begin{tabular}{lllll}
\hline $\begin{array}{l}\text { WHO } \\
\text { guidelines }\end{array}$ & \multicolumn{2}{l}{ Outcome } & \multirow{2}{*}{ Total } \\
\cline { 2 - 4 } & Discharged & Death & Referred & \\
\hline ASVS not given & 161 & 0 & 0 & 161 \\
Not followed & 606 & 16 & 3 & 625 \\
Followed & 132 & 13 & 4 & 149 \\
Total & 899 & 29 & 7 & 935 \\
\hline
\end{tabular}

ASVS: Anti-snake venom serum

The WHO, 2010 [1] and Haffkine Bio-Pharmaceuticals [14] give their guidelines to use ASVS on the basis of the quantity of snake venom injected in one bite depending on the species of the snake. The limitations to these guidelines are that the $\mathrm{WHO}$ and the manufacturers consider that the quantity of venom injected in one bite is the maximum capacity by the snake and do not consider phenomena such as dry bites, half bites, and intraspecies variations. Thus, when these guidelines are followed in the above snakebite cases, there might be chances of overuse of ASVS as less quantity of venom is injected into the patient. However, the production of ASVS is a long one and supply from the manufacturers is limited to government hospitals. Most of the cases of snakebite are reported to government hospitals and thus there is a high demand for ASVS in such centers. Due to the high demand and less supply, there is scarcity of ASVS in government hospitals. Thus, it is important that the treating physicians use ASVS optimally. We did not find any reported studies that showed utilization of ASVS in the past, due to which it was planned to carry out this study.

Majority cases of snakebite in this region were from the age groups of 15 to 59 years of age. The reason for this may be that the age group of 15-59 years is the age when people are employed in various occupations. The results of the present study match with a study conducted by Redewad et al., in 2014 [15], in central India, which stated that $52.60 \%$ cases were in the range of ages $21-40$ years. Similarly, Kirte et al., in 2002 [9], reported that $93 \%$ cases were from the age of 11 to 60 years. More males were exposed to snakebites may be because males are more involved in outdoor activities and jobs.

It is imperative that the snakebite patients are observed before administration of ASVS. However, many times ASVS are given without observing for signs of envenomation. This might be due to reasons like history of bite by dangerous snake or anxiety of patients. A few patients did not show any signs of envenomation, but still, ASVS was administered. The reason why there was unnecessary use of ASVS in these cases was not mentioned in the records. The probable reason for which ASVS was administered even when there were no signs of envenomation may be due to the panic and anxiety that is associated with snakebites. Furthermore, the patients or their relatives and sometimes even the physician may not want to take any chance because of medicolegal aspect of snakebite cases. Many a times in snakebite patients bite marks were not seen, but ASVS was administered. This might be because after bite many times the marks are not seen due to swelling of the local site. Furthermore, there might have been a history of dangerous bite or anxiety of patients and relatives [1]. Similarly, a few patients with bite marks were not given ASVS. The WHO states that before administration of ASVS, the patient has to be observed for signs of envenomation, for optimum use of ASVS [1]. The reason for not administration of ASVS in cases where bite marks were present could be due to the absence of signs of envenomation due to non-venomous snake or dry bites.

Looking in the number of vials consumed, we observed that majority of cases (615) were treated with 1-20 vials of ASVS during their stay at the tertiary center. If the WHO guidelines were strictly followed, then the number of vials required would be more than what we observed [1]. This might be probably because in most cases the snake was not identified and the treatment at the center was based on degree of envenomation. The average quantity of ASVS administered in cases with mild envenomation was 11.91 vials and in severe envenomation cases were 23.93 vials. In a study conducted by Syed Moied et al., [16] in 2012, the investigators found that the average dose of ASVS administered to patients with mild envenomation was $67 \mathrm{ml}$, moderate degree of envenomation was $107 \mathrm{ml}$, and severe cases were $196 \mathrm{ml}$. In our study, we found that the mean dose of ASVS in survived patients was $98.19 \mathrm{ml}$ and in death group was $186.20 \mathrm{ml}$. In a study conducted by Redewad et al., in 2014 [15], showed that the mean dose ASVS used in patients who survived snakebites was $310.98 \mathrm{ml}$ with a standard deviation of $297.39 \mathrm{ml}$. Similarly, patients who succumbed to snakebites received a mean dose of $392.66 \mathrm{ml}$ with a standard deviation of $275.81 \mathrm{ml}$

Singh, in 2010 [17], reported that in cases of snakebites $80-100 \mathrm{ml}$ of ASVS is to be administered for treatment. In a study conducted by Saini in North India, in 2014 [18], the authors have advocated the use of 8-10 vials, i.e. 80 to $100 \mathrm{ml}$ of ASVS as loading dose and a maximum of 30 vials and 20 vials for hemotoxic and neurotoxic patients, respectively. In a review article by Ray, in 2014 [19], the author has also stated the use of 80-100 ml ASVS for loading dose for the treatment of snakebites. In an observational study conducted by Agarwal, in 2005 [20], the investigators have compared the outcome of high-dose $(150 \mathrm{ml})$ and low-dose $(100 \mathrm{ml})$ ASVS in ventilator supported neuroparalytic snakebite patients. The investigators reported that there were no differences in the outcome in the two groups and concluded that the low-dose regimen was as good as the high-dose regimen. Furthermore, Srimannarayana et al. in their study, in 2004 [21], have tried to rationalize the use of ASVS. In their prospective interventional study, the investigators have randomized snakebite cases in three groups. Group I has standard dose regimen with $100 \mathrm{ml}$ loading dose and $50 \mathrm{ml} 6$ hourly, Group II has low dose $30 \mathrm{ml}$ loading dose and $30 \mathrm{ml}$ maintenance dose 6 hourly, and Group 3 was also low dose but with $70 \mathrm{ml}$ loading dose. The investigators concluded that the Group II and Group III regimens were equivalent for the treatment of mild and severe envenomation, respectively, as compared to standard regimen. They also commented that the low-dose regimen helped to save the much scarce ASVS.

In the present study, out of 625 cases where the WHO guidelines were not followed, 606 were discharged, 16 succumbed, and three were referred. Among 149 cases out of 935 where the WHO guidelines were followed, 132 were discharged, 13 died, and four were referred. Ghosh et al., in 2007 [22], concluded that there is a need to develop a nationally acceptable regimen for the use of ASVS to suit the local needs according to the locally prevalent snakes. There are protocols of the local institute that helps to cope with the need at local level, which may be very different than the global guidelines. In the current study, we observe that utilization of ASVS in 625 (80.74\%) cases was according to individualized regimen.

\section{CONCLUSION}

The guidelines issued by the WHO are based on the identification of the snake, degree of envenomation, and presence or absence of bite marks. In the present study, it was found that the identification of snake for treatment is difficult due to probable reasons such as darkness, lack of knowledge, sudden nature of the bite, panic, and anxiety of the of the patient and their relatives. Many a times due to anxiety and medicolegal implications and in a hurry to treat patient, the guidelines 
for ASVS dose are not followed. In view of the current situation, it is of utmost importance to use ASVS rationally and judiciously. Furthermore, many investigators have advocated the development of local regimen for optimum use of ASVS. We observed individualized treatment for treatment with ASVS, thereby reducing the use of ASVS. Thus, we conclude that individualized approach depending on locally predominant snakes for the treatment of snakebite based on the degree of envenomation is a way by which the scarce ASVS can be used optimally.

However, more region wise research is needed to formulate the policy for individualized treatment of snakebites. Local protocol and training are required to prepare doctors to improve treatment and reduce morbidity as well as mortality. It is recommended that effective ASVS usage protocol is developed and made a key priority by NHAs.

\section{LIMITATIONS}

The present study was conducted in a single institute. Multicentric studies in similar context would shade more light on the subject. We have collected data from only one institute; therefore, population is relatively homogenous. Hence, results cannot be extrapolated to general population.

\section{AUTHOR'S CONTRIBUTION}

All authors have contributed equally in developing the concept of study, data collection, data analysis, and drafting the manuscript.

\section{CONFLICTS OF INTEREST}

The authors declared that they have no conflicts of interest.

\section{REFERENCES}

1. Warrell DA. Guidelines for the Management of Snake-Bites. Geneva: WHO; 2010

2. Mohapatra B, Warrell DA, Suraweera W, Bhatia P, Dhingra N, Jotkar RM, et al. Snakebite mortality in India: A nationally representative mortality survey. PLoS Negl Trop Dis 2011;5:1-8.

3. Bhalla G, Mhaskar D, Agarwal A. A study of clinical profile of snakebite at a tertiary care center. Toxicol Int 2014;21:203-8.

4. Al-Mamun MA, Rahman MA, Hasan R, Rahmann Z, Haque KM. Histopathological alterations induced by common krait bungarus caeruleus venom on hepatic, Renal and cardiac tissues of albino mice. Int J Pharm Pharm Sci 2015;7:239-42.

5. Khan PA, Chary MD, Kumar MM. A study on treatment pattern and outcomes of poisoning cases in a tertiary care and government district hospital. Int J Pharm Pharm Sci 2017;9:193-7.

6. Inamdar K, Parhate SM, Randad RD. Pharmacotherapeutic study of efficacy, safety and prognostic analysis of anti snake venom serum in snake bite patients. Int J Basic Clin Pharmacol 2017;6:906-12.

7. Alirol E, Sharma SK, Bawaskar HS, Kuch U. Snake bite in south Asia : A review. PLoS Negl Trop Dis 2010;4:e603.

8. Punde DP. Management of snake-bite in rural Maharashtra: A 10-year experience. Natl Med J India 2005;18:71-5.

9. Kirte RC, Wahab SN, Bhathkule PR. Record based study of snake bite cases admitted at shri vasantrao naik government medical college and Hospital, Yavatmal (Maharashtra). Indian J Publc Heal 2002;25:12-4.

10. Whitaker R, Whitaker S. Venom, antivenom production and the medically important snakes of India. Curr Sci 2012;103:635-43.

11. Padilla A, Bon C, et al. WHO Guidelines for the Production Control and Regulation of Snake Antivenom Immunoglobulins. Geneva: WHO; 2008

12. Haffkine Biopharmaceuticals. Snake Antivenin. Haffkine Publication; 2016. Available from: http://www.fpnotebook.com/er/Pharm/SnkAntvn.htm.

13. Kalyan B, Nanda SS, Venkateshwarlu P, Kiran Y, Jadhav RT. Antisnake Venom Serum (ASVS). Int J Pharm Biomed Res 2010;1:76-89.

14. Haffkine Biopharmaceuticals, "Snake Antivenin," Haffkine Biopharmaceuticals;2018. Available from: http:/www.vaccinehaffkine. com/products/antitoxins-sera/snake-antivenin-detail.html

15. Redewad N, Bhaisare S, Bansod Y, Hire R. Management and outcome study of snake bite cases in central India. Sch J Appl Med Sci 2014;2:435-41

16. Ahmed SM, Nadeem A, Islam MS, Agarwal S, Singh L. Retrospective analysis of snake victims in Northern India admitted in a tertiary level institute. J Anaesthesiol Clin Pharmacol 2012;28:45-50.

17. Singh S, Singh G. Snake Bite: Indian Guidelines and Protocol. In: Text Book of Toxicology.; 2010. p. 424-6.

18. Saini V, Sardana D, Samra T. Management of snake bite victims in a Tertiary Care Intensive Care Unit in North India. Indian J Crit Care Med 2014;18:544-5.

19. Ray AS. Snake Bite its First Aid and Anti Snake Venom (ASV): Details Guidelines. Pharma Tutor 2014;2:85-8.

20. Agarwal R, Aggarwal AN, Gupta D, Behera D, Jindal SK. Low dose of snake antivenom is as effective as high dose in patients with severe neurotoxic snake envenoming. Emerg Med J 2005;22:397-401.

21. Srimannarayana J, Dutta TK, Sahai A, Badrinath S. Rational use of anti-snake venom (ASV): Trial of various regimens in hemotoxic snake envenomation. J Assoc Physicians India 2004;52:788-93.

22. Ghosh S, Maisnam I, Murmu BK, Mitra PK, Roy A, Simpson ID. Locally Developed Snakebite Management Protocol Significantly Reduces Overall Anti Snake Venom Utilization in West Bengal, India. Wilderness Environ Med 2008;19:267-74. 